\title{
MODELAGEM DO CRESCIMENTO E DA PRODUÇÃO DE Pinus taeda L. POR MEIO DE FUNÇÃO PROBABILÍSTICA
}

\author{
Rozane de Loyola Eisfeld*, Carlos Roberto Sanquetta**, Julio Eduardo Arce**, \\ Romualdo Maestri***, Karla Simone Weber**** \\ * Eng. Florestal, M. Sc., International Paper - rozane.eisfeld@ipaperbr.com \\ ** Eng. Florestal, Dr., Depto. de Ciências Florestais, UFPR - sanqueta@floresta.ufpr.br - jarce@floresta.ufpr.br \\ *** Eng. Florestal, Dr., Aracruz Celulose - rmaestri@aracruz.com.br \\ **** Eng. Florestal, Mestranda em Eng. Florestal, UFPR - karla@floresta.ufpr.br \\ Recebido para publicação: 08/11/2004 - Aceito para publicação: 14/06/2005
}

\begin{abstract}
Resumo
Modelagem do crescimento e da produção de Pinus taeda L. por meio de função probabilística. $\mathrm{O}$ presente trabalho teve como objetivo testar a função probabilística para a estimativa do crescimento e da produção. Para isso, foram utilizados dados de 325 parcelas permanentes de Pinus taeda L. sem desbaste, provenientes da empresa International Paper do Brasil. Foram ajustados os modelos de altura dominante, sítio, sobrevivência, área basal e variância dos diâmetros, e foi escolhida a distribuição Weibull para estimativa das distribuições diamétricas a partir do método dos momentos. Foram sorteadas 70 parcelas para se fazer a comparação entre os dados reais com os estimados. De modo geral, a função probabilística gerou resultados satisfatórios para estimativa do número de árvores, área basal e volume.

Palavras-chave: Simulação; projeção e predição; floresta plantada sem desbaste.
\end{abstract}

\section{Abstract}

Growth and yield modelling of Pinus taeda l. using probability function. The objective of this work was to test the "probability function" for growth and yield modelling. The data came from 325 permanent samples established in unthinned Pinus taeda L. (loblolly pine) stands owned by the International Paper of Brazil Co. It was used functions for dominant height, site, mortality, basal area and variance of the diameters. The Weibull probability distribution chosen to be used was fitted to data by the moments' method. Seventy sample plots were randomly chosen in order to make the comparison among the observed and predicted values. In general, the probability function provided satisfactory estimates for number of trees, basal area and stem volume per hectare.

Keywords: Simulation; projection and prediction; planted forests without thinning.

\section{INTRODUÇÃO}

O plantio de florestas no Brasil é uma atividade econômica importante e com grande impacto no campo social. Conforme Caron Neto (2001), o estado do Paraná possui a maior área de florestas plantadas de Pinus, totalizando 605.130 ha, ou seja, 33\% da área plantada total do Brasil. É indiscutível a importância desse gênero nesse Estado, que representa $90 \%$ de toda a madeira empregada na indústria, atingindo cerca de $20.000 .000 \mathrm{de} \mathrm{m}^{3}$ consumidos anualmente.

Face a isso, é do interesse de qualquer administrador ligado ao setor florestal conseguir quantificar e prognosticar, com confiabilidade, o estoque de suas florestas. A quantificação do crescimento e da produção consiste em uma condição essencial para definir a utilização dos bens advindos da floresta, além de fornecer informações que subsidiam a tomada de decisões para a maioria das atividades ligadas ao setor.

Uma alternativa possível para contornar esse problema é o emprego de uma ferramenta básica nas atividades de planejamento florestal, que é a técnica de modelagem do crescimento e da produção. Ao utilizar os sistemas de prognose do crescimento e produção, o profissional terá condições de definir antecipadamente o momento do desbaste, se este for um dos objetivos do empreendimento, a rotação 
econômica ótima, o planejamento da atividade de colheita e prescrever regimes de manejo adequados para cada espécie, em cada sítio, que visem à qualidade do produto final (Abreu, 2000).

O objetivo principal deste estudo foi ajustar modelos de crescimento e produção e testar a sua eficiência a partir da metodologia de função probabilística, utilizando dados de uma floresta de Pinus taeda sem desbaste.

\section{REVISÃO BIBLIOGRÁFICA}

A estrutura básica para o desenvolvimento de modelos de crescimento e produção a partir de função probabilística se dá na possibilidade de descrever a estrutura diamétrica das populações através de distribuições matemáticas definidas como função de densidade de probabilidade (fdp). As distribuições que se destacam são Gamma, Lognormal, Beta, SB Johnson, SBB de Johnson, Weibull, Exponencial e a Normal (Scolforo, 1998).

Para se estimar os parâmetros dessas distribuições, existem diferentes métodos, podendo-se destacar o método da máxima verossimilhança, o dos momentos e o dos percentis (Scolforo, 1998).

Em 1973, Bailey e Dell apresentaram a função Weibull aplicada à modelagem em florestas, considerando-a matematicamente simples e flexível e mais fácil de ser utilizada do que a distribuição Beta, pelo fato de não requerer integração numérica para o cálculo do número de indivíduos nas classes. Através dela, distribuições diamétricas decrescentes e unimodais foram ajustadas satisfatoriamente.

Maestri (1992) aplicou a função Weibull com três parâmetros para estimar a produção presente e futura de volume de madeira e peso de casca para povoamentos de acácia-negra. Para obter os parâmetros da função, ele utilizou a técnica de recuperação dos coeficientes "b" e "c", através de pares de diâmetros percentis, e do coeficiente "a" pela relação direta com o diâmetro mínimo da amostragem, obtendo resultados com aceitável grau de confiabilidade.

Abreu (2002), com dados precoces de Eucalyptus grandis, modelou os atributos da floresta (sítio, sobrevivência, relação hipsométrica genérica, diâmetro mínimo, diâmetro máximo, variância dos diâmetros, área basal e média aritmética dos diâmetros), ajustou e selecionou as funções probabilísticas como Beta, Weibull e SB por diferentes métodos e avaliou a veracidade das prognoses e a eficiência do modelo para realizá-las precocemente. Nesse estudo, foi verificado que a função de distribuição Weibull, ajustada pelo método dos momentos, mostrou-se a mais precisa.

As funções Beta, Gamma, Normal, SB de Johnson e Weibull com três parâmetros foram ajustadas e testadas por Valejos (2003) no desenvolvimento de um sistema de simulação de crescimento e produção de Populus spp. Com os resultados estatísticos favoráveis, ele implementou no sistema a função Weibull com três parâmetros, utilizando o método dos momentos para obtenção dos parâmetros.

Segundo Guimarães (1994), atualmente existe um consenso sobre a superioridade da função Weibull em relação às demais funções empregadas, quando aplicadas em amostras de distribuições diamétricas de florestas eqüiâneas. A explicação está no fato de as florestas eqüiâneas tenderem a apresentar distribuições assimétricas à direita, em razão das árvores maiores apresentarem maiores taxas de crescimento que as menores (efeito de competição), gerando uma configuração que coincide com as condições ideais para ajuste da função Weibull. Além do mais, a característica dessa função de descrever curvas com diferentes pontos de inflexão proporciona maior capacidade de ajuste em comparação com as demais funções, que em sua maioria apresentam curvatura rígida.

Já foram propostos muitos modelos para distribuição diamétrica, mas nenhum tem tantas características favoráveis quanto a distribuição Weibull. Os parâmetros provenientes dessa função proporcionam uma boa representação dos dados, assim como uma forte correlação entre as variáveis do povoamento. Facilidade na manipulação algébrica e habilidade de assumir uma variedade de formas de curvas fazem a função Weibull útil para os modelos biológicos (Bailey e Dell, 1973).

\section{MÉTODOS E MATERIAIS}

\section{Descrição da área de estudo}

Os dados de Pinus taeda utilizados para este estudo são provenientes das propriedades da empresa INTERNATIONAL PAPER DO BRASIL, localizada no município de Arapoti, no estado do Paraná. De acordo com a classificação de Koeppen, o clima da região é do tipo $\mathrm{Cfb}$, ou seja, subtropical quente temperado, caracterizado por apresentar temperatura média de $18{ }^{\circ} \mathrm{C}$ a $22^{\circ} \mathrm{C}$. (IAPAR, 2000). 


\section{Base de dados}

Foram utilizadas informações de 325 parcelas permanentes, com idades variando entre 4 e 25 anos, em condições variadas de sítio. Dessas, 172 parcelas continham duas medições, e 153 continham três medições.

Todas as parcelas são retangulares, com dimensões de $20 \times 30 \mathrm{~m}$, totalizando $600 \mathrm{~m}^{2}$ de área. Foram medidos todos as CAPs (circunferência à altura do peito) e algumas das alturas totais. Além disso, cada indivíduo foi codificado conforme o seu estado de sanidade (morta, torta, bifurcada, atacada por vespa).

Os povoamentos deste trabalho foram implantados em dois diferentes espaçamentos, 2,5 x 1,8 $\mathrm{m}$ e $3,0 \times 2,0 \mathrm{~m}$. Os mais jovens, entre 4 a 8 anos, foram plantados com o espaçamento maior, com um número inicial de 1.667 árvores. Já os povoamentos mais antigos, de 13 a 24 anos, foram plantados com 2.222 árvores.

Processamento das parcelas de inventário

- Altura

Foi utilizado o modelo modificado de Prodan, com a seguinte forma:

$h-1,3=\frac{D A P^{2}}{\beta_{0}+\beta_{1} D A P+\beta_{2} D A P^{2}+\beta_{3} D A P \times I d}$

Em que:

$h=$ Altura estimada (m);

$D A P=$ Diâmetro à altura do peito $(\mathrm{cm})$;

$I d=$ Idade do povoamento (anos);

$\beta_{o,} \beta_{1,} \beta_{2,} \beta_{3}=$ Parâmetros a serem estimados.

- Volume

Para se estimar o volume, foi utilizado o banco de cubagem fornecido pela empresa. Ao total, foi utilizada a cubagem rigorosa de 3.404 árvores de Pinus taeda, com idades variando de 1 a 24 anos, provenientes de todas as glebas da empresa.

A cubagem foi relativa, tomando-se medidas de diâmetro a 5\%, 10\%, 15\%, 25\%, 35\%, 45\%, 55\%, $65 \%, 75 \%, 85 \%$ e $95 \%$ da altura total das árvores. O cálculo dos volumes das seções foi obtido a partir da fórmula de Smalian.

A partir dessa base, foi possível ajustar o polinômio do $5^{\circ}$ grau para o cálculo do volume, que é assim representado:

$\frac{d i}{D A P}=\beta 0+\beta 1\left(\frac{h i}{h}\right)+\beta 2\left(\frac{h i}{h}\right)^{2}+\beta 3\left(\frac{h i}{h}\right)^{3}+\beta 4\left(\frac{h i}{h}\right)^{4}+\beta 5\left(\frac{h i}{h}\right)^{5}$

Em que:

$\beta_{s}=$ Parâmetros a serem estimados;

$d i=$ Diâmetro correspondente a uma altura hi $(\mathrm{cm})$;

$D A P=$ Diâmetro à $1,3 \mathrm{~m}$ de altura $(\mathrm{cm})$;

$h=$ Altura total $(\mathrm{m})$

$h i=$ Alturas obtidas nas diversas porcentagens da altura total $(\mathrm{m})$.

\section{Desenvolvimento dos modelos para a função probabilística}

Fase 1 - Classificação de sítio

As curvas de sítio foram geradas pelo método da diferença algébrica, avaliando o desempenho dos modelos na sua forma anamórfica.

Os dados foram ajustados em formato de predição, e os coeficientes obtidos foram utilizados também na projeção. 
Foram testadas as seguintes equações para expressar os índices de sítio:

a) Schumacher (1): $H_{d}=\beta_{0} \exp \left(-\beta_{1} \frac{1}{I d}\right)$
b) Schumacher (2): $\ln H_{d}=\beta_{0}+\beta_{1}\left(\frac{1}{I d}\right)^{\beta_{2}}$
c) Chapman-Richards: $H_{d}=\beta_{0}\left(1-\exp ^{-\beta_{1} \times I d}\right) \beta_{2}$
d) Bailey com três parâmetros: $H_{d}=\beta_{0}\left(1-\exp ^{-\beta_{1} \times I d^{\beta_{2}}}\right)$
e) Bailey com quatro parâmetros: $H_{d}=\beta_{0}\left(1-\exp -\beta_{1}^{\times I d} \beta_{2}\right)_{3}^{\beta_{3}}$

Em que:

$H_{d}=$ Altura média das 100 árvores mais grossas / ha (m);

$I d=$ Idade do povoamento (anos);

$e=$ Base do logaritmo natural;

$\beta_{o,} \beta_{1,} \beta_{2,} \beta_{3}=$ Parâmetros a serem estimados.

Fase 2 - Desenvolvimento de modelos dos atributos do povoamento

Para estimar os atributos do povoamento, foram ajustados e selecionados modelos entre os vários existentes na literatura florestal, para:

a) Altura dominante

Foram testados os modelos já descritos $(3,4,5,6$ e 7) .

b) Sobrevivência das árvores

Foram escolhidos os seguintes modelos a serem testados:

- Lenhart: $N_{2}=N_{1} \exp \left\{\beta_{1}\left(I d_{2}-I d_{1}\right)+\beta_{2}\left[\ln \left(\frac{I d_{2}}{I d_{1}}\right)\right]\right\}$

- $\quad$ Clutter: $N_{2}=N_{1}\left(I d_{2}-I d_{1}\right) \beta_{1} \exp \left[\left(\beta_{0}+\beta_{2} \times H d\right)\left(I d_{2}-I d_{1}\right)\right]$

- $\quad$ Pienaar e Shiver: $\ln N_{2}=\ln N_{1}-\beta_{1}\left(I_{2}^{\beta_{2}}-I_{1}^{\beta_{2}}\right)$

- Weibull: $N_{2}=N_{1}\left[\exp \frac{-\left(I d_{2}-I d_{1}\right)}{\beta_{1}}\right]^{\beta_{2}}$

- $\quad$ Silva: $N_{2}=N_{1} \exp \left(\beta_{0} \times \beta_{1}{ }^{I d_{2}}-\beta_{1}{ }^{I d_{1}}\right)$

- $\quad$ Modelo 1: $N_{2}=\left[N_{1} \times \beta_{1}+\beta_{2} \times\left(I d_{2} \times \beta_{3}-I d_{1} \times \beta_{3}\right)\right] \frac{1}{\beta_{1}}$

- $\quad$ Modelo 2: $N_{2}=\beta_{0}+\beta_{1} \times H d+\beta_{2} \times N_{1}\left(\frac{I d_{1}}{I d_{2}}\right)+\beta_{3} \times\left(1-\left(\frac{I d_{1}}{I d_{2}}\right)\right)$ 
Em que:

$N_{l}=$ Número de árvores $(\mathrm{N} / \mathrm{ha})$ na primeira medição;

$N_{2}=$ Número de árvores ( $\mathrm{N} / \mathrm{ha}$ ) na segunda medição;

$H d=$ Altura média das árvores dominantes $(\mathrm{m})$;

$I d_{1}=$ Idade do povoamento (anos) na primeira medição;

$I d_{2}=$ Idade do povoamento (anos) na segunda medição;

$\beta_{o}, \beta_{1,} \beta_{2}=$ Parâmetros a serem estimados.

c) Área basal

Foram testados na realização de uma predição:

- $\quad$ Modelo 1: $\ln G=\beta_{0}+\beta_{1}\left(\frac{1}{I d}\right)+\beta_{2} \ln (N)+\beta_{3} \ln (H d)$

- $\quad$ Modelo 2: $\ln G=\beta_{0}+\beta_{1}\left(\frac{1}{I d}\right)+\beta_{2} S+\beta_{3} \ln (N)$

- $\quad$ Modelo 3: $\ln G=\beta_{0}+\beta_{1} \ln N+\beta_{2} I+\beta_{3} \ln \left(\frac{1}{I d}\right)+\beta_{3} \ln \left(\frac{1}{S}\right)$

Os mesmos modelos foram utilizados, porém tratando-se de uma projeção:

- $\quad$ Modelo 1: $\ln G_{2}=\ln \left(G_{1}\right)+\beta_{1}\left(\frac{1}{I d_{2}}-\frac{1}{I d_{1}}\right)+\beta_{2}\left(\ln N_{2}-\ln N_{1}\right)+\beta_{3}\left(\ln H d_{2}-\ln H d_{1}\right)$

- $\quad$ Modelo 2: $\ln G_{2}=\ln \left(G_{1}\right)+\beta_{1}\left(\frac{1}{I d_{2}}-\frac{1}{I d_{1}}\right)+\beta_{2} S+\beta_{3}\left(\ln N_{2}-\ln N_{1}\right)$

- $\quad$ Modelo 3:

$$
\ln G_{2}=\ln \left(G_{1}\right)+\beta_{1}\left(\ln N_{2}-\ln N_{1}\right)+\beta_{2}\left(I d_{2}-I d_{1}\right)+\beta_{3}\left(\ln \frac{1}{I d_{2}}\right)-\left(\ln \frac{1}{I d_{1}}\right)+\beta_{3} \ln \left(\frac{1}{S}\right)
$$

Em que:

$G_{l}=$ Área basal $\left(\mathrm{m}^{2} / \mathrm{ha}\right)$ na primeira medição;

$G_{2}=$ Área basal $\left(\mathrm{m}^{2} / \mathrm{ha}\right)$ na segunda medição;

$N=$ Número de árvores $(\mathrm{N} / \mathrm{ha})$;

$S=$ Índice de sítio $(\mathrm{m})$;

$I d=$ Idade do povoamento (anos);

$H d_{l}=$ Altura média das árvores dominantes $(\mathrm{m})$ na primeira medição;

$\mathrm{Hd}_{2}=$ Altura média das árvores dominantes (m) na segunda medição;

$\beta_{o,} \beta_{1,} \beta_{2,} \beta_{3}=$ Parâmetros a serem estimados.

d) Variância dos diâmetros:

Foram testados na realização de uma predição:

- $\quad \operatorname{Maestri}(1): \ln \left(S^{2} d\right)=\beta_{0}+\beta_{1} \ln \left(\frac{N}{G}\right)+\beta_{2} H d^{-1}+\beta_{3} I d^{2}$

- $\quad$ Maestri (2): $\ln \left(S^{2} d\right)=\beta_{0}+\beta_{1} H d+\beta_{2} \ln (N)+\beta_{3} \ln (I d)$

- $\quad$ Knoebell: $\ln \left(S^{2} d\right)=\beta_{0}+\beta_{1} \log (G)+\beta_{2} \log (H d)+\beta_{3} \ln (\operatorname{Id} \times N)$

- Scolforo: $\ln \left(S^{2} d\right)=\beta_{0}+\beta_{1} \log (H d)+\beta_{2} \log \left(\frac{G}{N}\right)+\beta_{3}\left(\frac{H d}{I d}\right)$ 
Para realizar uma projeção, foram testados os seguintes modelos:

- $\quad$ Modelo 1: $\ln \left(S^{2} d_{2}\right)=\beta_{1} S^{2} d_{1}+\beta_{2} D g_{1}+\beta_{3} \frac{N_{1}}{N_{2}}$

- $\quad$ Modelo 2: $\ln \left(S^{2} d_{2}\right)=\beta_{1} D m_{1}+\beta_{2} D g_{1}+\beta_{3} \frac{I d_{1}}{I d_{2}}+\beta_{4}\left(\ln N_{2}-\ln N_{1}\right)+\beta_{5}\left(\ln G_{2}-\ln G_{1}\right)+\beta_{6}\left(\ln H d_{2}-\ln H d_{1}\right)$

- $\quad$ Modelo 3: $S^{2} d_{2}=\beta_{1} S^{2} d_{1}+\beta_{2} D g_{1}+\beta_{3} \frac{N_{1}}{N_{2}}$

Em que:

$S^{2} d_{1}=$ Variância dos diâmetros $\left(\mathrm{cm}^{2}\right)$ na primeira medição;

$S^{2} d_{2}=$ Variância dos diâmetros $\left(\mathrm{cm}^{2}\right)$ na segunda medição;

$D g=$ Diâmetro médio quadrático $(\mathrm{cm})$;

$D m=$ Média aritmética dos diâmetros $(\mathrm{cm})$;

$G=$ Área basal $\left(\mathrm{m}^{2} / \mathrm{ha}\right)$;

$N=$ Número de árvores $(\mathrm{N} / \mathrm{ha})$;

$I d=$ Idade do povoamento (anos);

$H d=$ Altura média das árvores dominantes $(\mathrm{m})$;

$\beta_{o}, \beta_{1,} \beta_{2}, \beta_{3}=$ Parâmetros a serem estimados.

O resultado obtido no modelo ajustado foi necessário para o cálculo do parâmetro "c", na distribuição de Weibull.

Fase 3 - Ajuste da distribuição diamétrica

Devido às suas propriedades estatísticas, aplicações práticas e ao sucesso que essa distribuição obteve em diversos trabalhos com diferentes espécies, a distribuição Weibull foi escolhida para ser testada na modelagem da distribuição diamétrica.

A distribuição Weibull de três parâmetros tem sua função de densidade de probabilidade (fdp) descrita como:

$f(x)=\frac{c}{b}\left(\frac{X-a}{b}\right)^{c-1} \exp \left[-\left(\frac{X-a}{b}\right)^{c}\right]$

Em que:

$a=$ Parâmetro de locação;

$b=$ Parâmetro de escala;

$c=$ Parâmetro de forma;

$X=$ Variável de interesse (diâmetro).

O parâmetro "a" representa o menor limite da distribuição. A distribuição Weibull é uma função flexível e pode assumir praticamente todas as formas que uma distribuição diamétrica necessita.

Existem diferentes formas para ajustar a distribuição Weibull, porém foi testado apenas o método dos momentos. A partir desse método, os parâmetros são estimados pelas seguintes equações:

CVest $=\frac{s d}{d}=\frac{\sqrt{\Gamma\left(1+\frac{2}{c}\right)-\Gamma^{2}\left(1+\frac{1}{c}\right)}}{\Gamma\left(1+\frac{1}{c}\right)}$

Em que:

$C V=$ Coeficiente de variação em diâmetro;

$s d=$ Desvio padrão do diâmetro ou raiz quadrada da variância do diâmetro;

$d=$ Diâmetro médio aritmético do povoamento $(\mathrm{cm})$;

$c=$ Parâmetro de forma;

$\Gamma=$ Função gama. 
Tendo-se o coeficiente de variação, o "c" pode ser calculado por um processo interativo.

Obtido o "c", "b" pode ser estimado como:

$b=\frac{d}{\Gamma\left(1+\frac{1}{c}\right)}$

Em que:

$d=$ Diâmetro médio;

$\Gamma=$ Função gama;

$c=$ Parâmetro "c" estimado.

O parâmetro "a" pode ser obtido independentemente. Com isso, seu valor é determinado dentro do processo de compatibilização da área basal advinda da distribuição (soma das áreas transversais de cada classe diamétrica) com a área basal advinda do modelo.

Fase 4-Modelo hipsométrico e função para estimativa do volume

Foi utilizado o modelo hipsométrico de Prodan para a estimativa da altura e o modelo polinomial não-segmentado do $5^{\circ}$ grau para a estimativa de volume, ambos já descritos anteriormente.

\section{Fase 5 - Prognose do crescimento e da produção}

Para a obtenção da prognose do crescimento e da produção do volume por classe diamétrica para o povoamento de Pinus taeda, os atributos do povoamento são prognosticados para a idade em que se deseja a projeção do volume, e os parâmetros da distribuição selecionados são então estimados. Com esses parâmetros estimados, pode-se encontrar a probabilidade de as árvores ocorrerem em cada classe diamétrica.

O produto dessa probabilidade pelo número de árvores sobreviventes permite estimar o número de árvores em cada classe diamétrica. Pode-se, então, utilizando a equação genérica selecionada, estimar a altura correspondente a cada centro de classe diamétrica. Assim, com o diâmetro e a altura, o volume foi estimado e extrapolado por hectare, ao se efetuar o seu produto pelo número de árvores.

\section{Critérios de seleção dos modelos ajustados}

Para testar a qualidade dos ajustes dos modelos, foram utilizados três parâmetros de comparação: o coeficiente de determinação $\left(\mathrm{R}^{2}\right)$, o erro padrão da estimativa (Syx) e a análise gráfica dos resíduos.

\section{Precisão da prognose e comparação entre modelos}

Para a avaliação da precisão da prognose, foram confrontados dados de 70 parcelas escolhidas aleatoriamente em diferentes idades, com a freqüência, a área basal e o volume prognosticado a partir da metodologia da função probabilística.

\section{RESULTADOS}

\section{Fase 1 - Classificação de sítio}

Foram testados diferentes modelos para classificar o sítio, e as estatísticas utilizadas na escolha estão apresentadas na Tabela 1.

Com base nessas estatísticas, foi escolhido o modelo de Bailey com 4 parâmetros. A equação ajustada ficou com a seguinte forma:

$$
H d=26,9462\left[1-\exp \left(-0,0011 \times I d^{2,613}\right)\right]^{0,4287}
$$

A equação descrita (31) é utilizada em uma predição. Esses mesmos coeficientes poderão também ser empregados na projeção. Nesse caso, o modelo terá a seguinte formulação: 
$\left.H d_{2}=H d_{1} \times\left\{1-\exp \left(-0,0011 \times I d_{2}^{2,613}\right)\right]\left[1-\exp \left(-0,0011 \times I d_{1}^{2,613}\right)\right]\right\}^{0,4287}$

Tabela 1. Estatísticas para comparar os modelos de sítio.

Table 1. Statistics to compare the site models.

\begin{tabular}{lccc}
\hline Modelo & Syx (m) & Syx (\%) & $\mathbf{R}^{\mathbf{2}}$ \\
\hline Schumacher 1 & 1,48078 & 7,8 & $96,41 \%$ \\
Schumacher 2 & 1,46385 & 7,7 & $96,49 \%$ \\
Chapman e Richards & 1,34296 & 7,1 & $97,05 \%$ \\
Bailey 3 parâmetros & 1,30016 & 6,9 & $97,23 \%$ \\
Bailey 4 parâmetros & 1,23943 & 6,5 & $97,49 \%$ \\
\hline
\end{tabular}

A Tabela 2 mostra os resultados dos coeficientes obtidos em cada um dos modelos testados.

Tabela 2. Coeficientes obtidos nos modelos de altura dominante.

Table 2. Obtained coefficients in the dominant height models.

\begin{tabular}{lcccc}
\hline Modelo & b0 & b1 & b2 & b3 \\
\hline Schumacher 1 & 37,281 & 7,352 & - & - \\
Schumacher 2 & 3,690 & 2,584 & - & - \\
Chapman e Richards & 28,593 & 0,151 & 1,882 & - \\
Bailey 3 parâmetros & 27,564 & 0,034 & 1,492 & - \\
Bailey 4 parâmetros & 26,946 & 0,0011 & 2,613 & 0,429 \\
\hline
\end{tabular}

Adotou-se como Idade Índice ou Idade de Referência o ponto aos 15 anos, uma vez que este se aproxima mais da idade de rotação silvicultural utilizada na empresa e também porque nessa idade é possível vislumbrar com mais propriedade o comportamento do sítio. e superior.

Foram definidas 5 classes de sítio, sintetizadas na Tabela 3, sendo estipulados os limites inferior

Tabela 3. Limites inferiores e superiores para cada classe com seu respectivo índice de sítio.

Table 3. Inferior and superior limits to each class with its respective site index.

\begin{tabular}{lccc}
\hline Classes & Limite Inferior $(\mathbf{m})$ & Limite Superior $(\mathbf{m})$ & IS (15 anos) \\
\hline Classe I & 26,1 & 27,7 & 26,9 \\
Classe II & 24,5 & 26,1 & 25,3 \\
Classe III & 22,9 & 24,5 & 23,7 \\
Classe IV & 21,3 & 22,9 & 22,1 \\
Classe V & 19,7 & 21,3 & 20,5 \\
\hline
\end{tabular}

\section{Fase 2 - Modelos do povoamento}

a) Altura dominante:

Como já descrito anteriormente, o modelo escolhido para o ajuste da altura dominante foi o modelo (31) de Bailey com 4 parâmetros.

b) Sobrevivência das árvores:

Foram testados sete diferentes modelos, ajustados na forma linear e não linear, para estimar satisfatoriamente a sobrevivência das árvores. As estatísticas utilizadas na escolha estão apresentadas na Tabela 4.

Apesar de o modelo de Clutter apresentar as melhores estatísticas, apenas o modelo de Silva e o modelo de Pienaar eram compatíveis, ou seja, partindo-se de diferentes idades, chega-se ao mesmo valor 
de número de árvores. Portanto, como o modelo de Silva foi superior ao modelo de Pienaar, com coeficiente de determinação de $98,78 \%$, erro padrão da estimativa de $1,94 \%$ (27 árvores por hectare) e resíduos sem tendenciosidades, ele foi escolhido para ser utilizado, estando representado na equação (34).

Tabela 4. Estatísticas para comparar os modelos de sobrevivência.

Table 4. Statistics to compare the survival models.

\begin{tabular}{lccc}
\hline Modelos & Syx (N/ha) & Syx (\%) & $\mathbf{R}^{\mathbf{2}}$ \\
\hline Lenhart & 21,6467 & 1,52 & $99,25 \%$ \\
Clutter & 20,9018 & 1,47 & $99,30 \%$ \\
Pienaar & 33,4595 & 2,35 & $98,20 \%$ \\
Weibull & 23,6471 & 1,66 & $99,10 \%$ \\
Silva & 27,5694 & 1,94 & $98,78 \%$ \\
Modelo 1 & 26,3278 & 1,85 & $98,89 \%$ \\
Modelo 2 & 26,2627 & 1,85 & $98,89 \%$ \\
\hline
\end{tabular}

$N_{2}=N_{1} \times \exp \left[76,16091 \times\left(0,999835^{I d_{2}}-0,999835^{I d}\right)\right]$

Em se tratando de uma predição, foi considerada uma mortalidade de 5\% no primeiro ano de plantio, e nos anos seguintes empregou-se a equação (34).

A Tabela 5 mostra os resultados dos coeficientes obtidos em cada um dos modelos testados.

Tabela 5. Coeficientes obtidos nos modelos de sobrevivência.

Table 5. Obtained coefficients in the survival models.

\begin{tabular}{lcccc}
\hline Modelo & b0 & b1 & b2 & b3 \\
\hline Lenhart & - & $-0,0321$ & 0,1666 & - \\
Clutter & 0,0008 & 0,0068 & $-0,0012$ & - \\
Pienaar & - & 0,0003 & 2,1848 & - \\
Weibull & - & 0,5785 & 0,0004 & - \\
Silva & 76,1609 & 0,9998 & - & - \\
Modelo 1 & - & 0,9992 & $-6,2155$ & 3,8773 \\
Modelo 2 & 13,7232 & $-3,6114$ & 1,0264 & 1486,0261 \\
\hline
\end{tabular}

c) Área basal:

As estatísticas na escolha do melhor modelo para a área basal estão apresentadas nas Tabelas 6 e 7.

Tabela 6. Estatísticas para comparar os modelos para a área basal utilizando uma predição.

Table 6. Statistics to compare the basal area models using prediction.

\begin{tabular}{lccc}
\hline Modelos de Predição & Syx $\left(\mathbf{m}^{\mathbf{2}} / \mathbf{h a}\right)$ & Syx $(\%)$ & $\mathbf{R}^{\mathbf{2}}$ \\
\hline Modelo 1 & 5,25060 & 11,99 & $87,47 \%$ \\
Modelo 2 & 5,06397 & 11,56 & $88,34 \%$ \\
Modelo 3 & 5,35420 & 12,22 & $86,97 \%$ \\
\hline
\end{tabular}

Na predição, o segundo modelo, que considera a idade, o sítio e o número de árvores como variáveis independentes, obteve as melhores estatísticas de ajuste com o maior coeficiente de determinação $(88,34 \%)$, o menor erro padrão da estimativa $(11,56 \%)$ e resíduos sem tendenciosidades. A equação ficou assim ajustada:

$\ln G=-1,84993-6,92813\left(\frac{1}{I}\right)+0,087323 \times S+0,586097 \times \ln (N)$ 
Tabela 7. Estatísticas para comparar os modelos para a área basal em se tratando de uma projeção. Table 7. Statistics to compare the basal area models using projection.

\begin{tabular}{|c|c|c|c|}
\hline Modelos de Projeção & $\operatorname{Syx}\left(\mathrm{m}^{2} / \mathrm{ha}\right)$ & Syx (\%) & $\mathbf{R}^{2}$ \\
\hline Modelo 1 & 1,54437 & 3,31 & $98,62 \%$ \\
\hline Modelo 2 & 1,57284 & 3,37 & $98,57 \%$ \\
\hline Modelo 3 & 1,75022 & 3,75 & $98,21 \%$ \\
\hline
\end{tabular}

Já na projeção, o primeiro modelo, que estima a área basal em função da idade, do número de árvores, da altura dominante e da área basal em um período inicial, apresentou o maior coeficiente de determinação $(98,62 \%)$ e o menor erro padrão da estimativa, variando em $1,54 \mathrm{~m}^{2} /$ ha correspondendo a 3,31\%. Portanto, esse modelo foi o escolhido, possuindo a seguinte formulação:

$$
\ln G_{2}=\ln \left(G_{1}\right)-4,78032 \times\left(\frac{1}{I_{2}}-\frac{1}{I_{1}}\right)+0,653767 \times\left(\ln N_{2}-\ln N_{1}\right)+0,51379 \times\left(\ln H d_{2}-\ln H d_{1}\right)
$$

d) Variância dos diâmetros:

As estatísticas utilizadas na escolha do melhor modelo para a variância dos diâmetros estão apresentadas nas tabelas 8 e 9 .

Tabela 8. Estatísticas para comparar os modelos de variância dos diâmetros em uma predição.

Table 8. Statistics to compare the diameter variance models in one prediction.

\begin{tabular}{lccc}
\hline Modelos de Predição & Syx $\left(\mathbf{c m}^{\mathbf{2}}\right)$ & Syx $\mathbf{( \% )}$ & $\mathbf{R}^{\mathbf{2}}$ \\
\hline Maestri 1 & 4,65526 & 27,97 & $76,98 \%$ \\
Maestri 2 & 4,87794 & 29,30 & $74,73 \%$ \\
Knoebell & 4,44856 & 26,72 & $78,98 \%$ \\
Scolforo & 4,69629 & 28,21 & $76,57 \%$ \\
\hline
\end{tabular}

Tabela 9. Estatísticas para comparar os modelos de variância dos diâmetros em uma projeção. Table 9. Statistics to compare the diameter variance models in one projection.

\begin{tabular}{lccc}
\hline Modelos de Projeção & Syx $\left(\mathbf{c m}^{\mathbf{2}}\right)$ & Syx $(\mathbf{\%})$ & $\mathbf{R}^{\mathbf{2}}$ \\
\hline Modelo 1 & 5,02751 & 27,89 & $74,27 \%$ \\
Modelo 2 & 3,65911 & 20,30 & $86,37 \%$ \\
Modelo 3 & 1,45668 & 8,08 & $97,84 \%$ \\
\hline
\end{tabular}

Na predição, o modelo de Knoebell obteve as melhores estatísticas de ajuste com o maior coeficiente de determinação $(78,98 \%)$, o menor erro padrão da estimativa $(26,72 \%)$ e resíduos sem tendenciosidades. A equação ficou assim ajustada:

$$
\ln \left(S^{2} d\right)=4,56912+0,90924 \log (G)+4,23082 \log (H d)-0,88773 \ln (\operatorname{Id} \times N)
$$

Nota-se que para a predição todos os modelos alcançaram erros acima de $27 \%$, evidenciando a dificuldade em se ajustar satisfatoriamente um modelo para a variância dos diâmetros quando se tem apenas informação inicial do povoamento.

Já na projeção, foi escolhido o modelo 3, com um coeficiente de determinação de 97,84\% e um erro padrão da estimativa de $8,08 \%$, variando em $1,45 \mathrm{~cm}^{2}$. O modelo escolhido ficou com a seguinte formulação:

$$
S^{2} d_{2}=1,0134 \times S^{2} d_{1}-0,1720 \times d g_{1}+4,7507 \times \frac{N_{1}}{N_{2}}
$$


Fase 3 - Ajuste da distribuição diamétrica

Cada projeção realizada resulta em diferentes valores de "a", "b" e "c", variando conforme as variáveis do povoamento.

\section{Fase 4 - Modelo hipsométrico e função para estimativa de volume}

Foram ajustados três modelos hipsométricos de Prodan, um para cada classe de idade, como descrito na Tabela 10.

Tabela 10. Coeficientes para o modelo hipsométrico modificado de Prodan por classe de idade.

Table 10. Coefficients for hipsometric modified model of Prodan by age class.

\begin{tabular}{lccccc}
\hline \multirow{2}{*}{ Classe de Idade } & b0 & b1 & b2 & b3 & b4 \\
\cline { 2 - 6 } & 0,904619 & 2,438855 & 0,044411 & $-0,209688$ & 0,904619 \\
\hline$<10$ anos & 1,302172 & 0,434440 & 0,028416 & $-0,005210$ & 1,302172 \\
$10-25$ anos & 4,430364 & 0,213686 & 0,032653 & $-0,002505$ & 4,430364 \\
\hline 25 anos & & & &
\end{tabular}

Para o volume, foram utilizados dados de cubagem de 3.404 árvores, ajustando-se o modelo polinomial do $5^{\circ}$ grau por classe de altura. Os coeficientes ajustados para cada classe estão relacionados na Tabela 11.

Tabela 11. Coeficientes para o modelo polinomial do $5^{\circ}$ grau nas diferentes classes de altura.

Table 11. Coefficient for polinomial model of fifth grade in different classes of height.

\begin{tabular}{lcccccc}
\hline Classe de & \multicolumn{7}{c}{ Coeficientes } \\
\cline { 2 - 7 } Altura & b0 & b1 & b2 & b3 & b4 & b5 \\
\hline$<9,9 \mathrm{~m}$ & 1,34327 & $-3,07285$ & 9,25382 & $-19,37126$ & 17,99750 & $-6,05783$ \\
$10-19,9 \mathrm{~m}$ & 1,16006 & $-2,21479$ & 6,36350 & $-11,95738$ & 9,24788 & $-2,54230$ \\
$20-29,9 \mathrm{~m}$ & 1,08987 & $-1,78586$ & 4,73138 & $-7,35889$ & 3,69926 & $-0,33847$ \\
$>30 \mathrm{~m}$ & 1,03315 & $-1,34179$ & 2,66713 & $-2,91480$ & $-0,32721$ & 0,89625 \\
\hline
\end{tabular}

\section{Fase 5 - Prognose do crescimento e da produção}

O volume total, a área basal e o número de árvores foram calculados a partir da freqüência real por classe diamétrica e comparados com a freqüência obtida na função probabilística, para 70 parcelas escolhidas aleatoriamente. Esses resultados foram analisados e são exibidos a seguir.

A Tabela 12 apresenta o erro obtido, por idade, na prognose do número de árvores, área basal e volume, com base nas 70 parcelas analisadas.

Tabela 12. Estatísticas na prognose do número de árvores, área basal e volume.

Table 12. Statistics in the simulation of number of tress, basal area and stem volume.

\begin{tabular}{ccccccc}
\hline \multirow{2}{*}{ Idade } & \multicolumn{2}{c}{ Número de árvores } & \multicolumn{2}{c}{ Área basal } & \multicolumn{2}{c}{ Volume } \\
\cline { 2 - 6 } & $\mathbf{S y x}$ & $\mathbf{R}^{\mathbf{2}}$ & $\mathbf{S y x}$ & $\mathbf{R}^{\mathbf{2}}$ & $\mathbf{S y \mathbf { x }}$ & $\mathbf{R}^{\mathbf{2}}$ \\
\hline Todas & $2,52 \%$ & $98,18 \%$ & $7,42 \%$ & $93,20 \%$ & $3,42 \%$ & $99,61 \%$ \\
4 & $1,48 \%$ & $91,46 \%$ & $48,31 \%$ & $34,56 \%$ & $16,64 \%$ & $49,11 \%$ \\
5 & $1,50 \%$ & $95,59 \%$ & $12,43 \%$ & $43,78 \%$ & $8,39 \%$ & $82,70 \%$ \\
6 & $1,55 \%$ & $94,76 \%$ & $5,64 \%$ & $92,20 \%$ & $6,76 \%$ & $92,36 \%$ \\
13 & $3,75 \%$ & $55,57 \%$ & $5,07 \%$ & $55,57 \%$ & $5,46 \%$ & $55,57 \%$ \\
18 & $3,08 \%$ & $91,20 \%$ & $2,54 \%$ & $89,47 \%$ & $2,50 \%$ & $91,31 \%$ \\
19 & $3,60 \%$ & $89,74 \%$ & $2,95 \%$ & $93,00 \%$ & $3,05 \%$ & $95,28 \%$ \\
20 & $3,05 \%$ & $90,31 \%$ & $2,22 \%$ & $89,82 \%$ & $2,26 \%$ & $91,14 \%$ \\
21 & $3,67 \%$ & $96,12 \%$ & $1,94 \%$ & $92,03 \%$ & $1,98 \%$ & $92,33 \%$ \\
\hline
\end{tabular}


A metodologia empregada estimou bem o número total de árvores. O maior erro de estimativa foi de 3,75\% aos 13 anos. Para todas as parcelas, o erro obtido na distribuição Weibull foi de 2,52\%, correspondendo a 35 árvores por hectare. $\mathrm{O}$ erro aumenta nas idades mais avançadas, em que a mortalidade é pouco influenciada pelo diâmetro e difícil de ser modelada.

Para a área basal, o erro médio foi de $3,54 \mathrm{~m}^{2} /$ ha, diminuindo com o aumento da idade.

Houve uma boa estimativa do volume total, em que o erro na estimativa foi de $3,42 \%$, o que corresponde a $15,51 \mathrm{~m}^{3} /$ ha. Nota-se que há uma diminuição no erro à medida que aumentam as idades. Os povoamentos mais jovens possuem uma grande variabilidade de crescimento, o que vem a prejudicar as estimativas e aumentar o erro padrão. Já povoamentos um pouco mais velhos, com 19 ou 20 anos, por exemplo, possuem erros bem inferiores, projetando o volume com mais precisão.

\section{CONCLUSÕES}

- As equações selecionadas para os atributos do povoamento se apresentaram de forma eficiente, proporcionando estimativas precisas.

- O erro médio obtido na simulação do número de árvores foi em torno de 2,5\%.Para a área basal, o erro ficou em torno de 7,4\%, diminuindo com o aumento da idade. $O$ volume obteve bons resultados, com erro na estimativa de 3,4\%.A metodologia utilizada se mostrou eficiente na estimativa do crescimento e da produção.O estudo contínuo dessa metodologia se mostra imprescindível na melhora contínua de uma metodologia consagrada, eficiente e amplamente difundida no Brasil.

\section{REFERÊNCIAS}

ABREU, E. C. R. Modelagem para prognose precoce do volume por classe diamétrica para Eucalyptus grandis. Lavras, 2000. 69f. Dissertação (Mestrado em Ciências Florestais), Universidade Federal de Lavras.

ABREU, E. C. R.; SCOlfORO, J. R. S.; OliveIRA, A. D.; MEllO, J. M.; KANEGAE, H. J. Modelagem para prognose precoce do volume por classe diamétrica para Eucalyptus grandis. Revista Scientia Forestalis, Piracicaba, n.61, p.86-102, jun, 2002.

BAILEY, R.L.; DELL, T.R. Quantifying diameters distributions with the Weibull functions. Forest Science, Washington, v.19, n.2, p.27-104, june, 1973.

CARON NETO, M. Pinus conquista espaço na região Sul. Revista da Madeira, Curitiba, n.58, v.10, p.24-28, 2001.

GUIMARÃES, D. P. Desenvolvimento de um modelo de distribuição diamétrica de passo invariante para prognose e projeção da estrutura de povoamentos de eucalipto. Viçosa, MG, 1994. 138f. Tese (Doutorado em Ciências Florestais), Universidade Federal de Viçosa.

IAPAR. Cartas climáticas do Estado do Paraná 1.0. Ponta Grossa, 2000. 1 CD - ROM.

MAESTRI R. Estimativa de produção presente e futura de volume de madeira e peso de casca para povoamentos de Acácia - Negra (Acacia mearnsii de Wild). Curitiba, 1992. 102f. Dissertação (Mestrado em Ciências Florestais) Setor de Ciências Agrárias, Universidade Federal do Paraná.

SCOLFORO, J. R. S. Modelagem do crescimento e da produção de florestas plantadas e nativas. Lavras: UFLA/FAEPE, 1998, 441p.

VALEJOS, O. S. B. Sistema de simulação de crescimento e produção de Populus spp., no Chile (Sistema SALICA). Curitiba, 2003. 110f. Tese (Doutorado em Ciências Florestais) Setor de Ciências Agrárias, Universidade Federal do Paraná. 